\title{
On the proportion of cancer stem cells in a tumour
}

\author{
Matthew D. Johnston a,b, Philip K. Maini a,c,*, S. Jonathan Chapman ${ }^{\mathrm{b}}$, \\ Carina M. Edwards ${ }^{\mathrm{d}}$, Walter F. Bodmer ${ }^{\mathrm{e}}$ \\ a Centre for Mathematical Biology, Mathematical Institute, University of Oxford, 24-29 St. Giles', Oxford OX1 3LB, UK \\ ${ }^{\mathrm{b}}$ Oxford Centre for Industrial and Applied Mathematics, Mathematical Institute, University of Oxford, 24-29 St. Giles', Oxford OX1 3LB, UK \\ c Oxford Centre for Integrative Systems Biology, Department of Biochemistry, University of Oxford, South Parks Road, Oxford OX1 3QU, UK

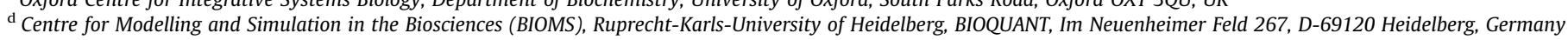 \\ e Cancer and Immunogenetics Laboratory, Cancer Research UK, Weatherall Institute of Molecular Medicine, John Radcliffe Hospital, Oxford OX3 9DS, UK
}

\section{A R T I C L E I N F O}

Article history:

Received 22 April 2010

Received in revised form

13 July 2010

Accepted 24 July 2010

Available online 3 August 2010

Keywords:

Cancer progression

Mathematical model

Feedback

\begin{abstract}
A B S T R A C T
It is now generally accepted that cancers contain a sub-population, the cancer stem cells (CSCs), which initiate and drive a tumour's growth. At least until recently it has been widely assumed that only a small proportion of the cells in a tumour are CSCs. Here we use a mathematical model, supported by experimental evidence, to show that such an assumption is unwarranted. We show that CSCs may comprise any possible proportion of the tumour, and that the higher the proportion the more aggressive the tumour is likely to be.
\end{abstract}

(c) 2010 Elsevier Ltd. All rights reserved.
Cancer stem cells are the cells that both initiate and propel cancer through all its many phases of growth (Reya et al., 2001; McDonald et al., 2006; Polyak and Hahn 2006). They, in general, have the ability to differentiate into a variety of cell types according to the tissue of origin (Mackenzie 2005; Locke et al., 2005; Harper et al., 2007). A cancer, therefore, to a greater or lesser extent recapitulates the organisation of the normal tissue from which it is derived, but in a deranged manner. Cancer therapy cannot be successful unless it eliminates the CSCs. Thus, understanding the nature and evolution of stem cells in a cancer is crucially important for the development of effective therapies.

A variety of approaches, especially selecting for certain surface markers using monoclonal antibodies and fluorescence activated cell sorter (FACS) technology, have been applied to the identification of CSCs in a wide range of cancers and cancer derived cell lines (see, for example, Al-Hajj et al., 2003; Singh et al., 2004; O'Brien et al., 2007; Ricci-Vitiani et al., 2007; Cho and Clarke 2008; Yeung et al., 2010). In many cases it has, however, been assumed that CSCs necessarily constitute only a small minority of the cells in a cancer (see, for example, O'Brien et al., 2007; Ricci-Vitiani et al., 2007; Dalerba et al., 2007a, 2007b; Clarke 2004). This assumption has largely been based on data suggesting that only a minority of

\footnotetext{
* Corresponding author at: Centre for Mathematical Biology, Mathematical Institute, University of Oxford, 24-29 St. Giles', Oxford OX1 3LB, UK.

E-mail address: maini@maths.ox.ac.uk (P.K. Maini).
}

the cancer cells xenografted from any given human cancer into immunodeficient mice can form a tumour. Modelling has also been used to support the idea that cancer stem cells are comparatively rare in a cancer (Huntly and Gilliland, 2005; Michor et al., 2005). However, more recent work on melanomas by Quintana et al. (2008) has shown that, while earlier techniques could identify only one in 837000 cells in a melanoma as being cancer stem cells, their newer assay suggested that as many as one in four cells from melanomas could form tumours in more stringently immunodeprived mice and observed over a longer period of time (see also Baker, 2008). Previous studies on mouse leukaemias have also shown that at least some malignancies may be maintained by more than $10 \%$ of the cells in a tumour (Kelly et al., 2007). Recently, Yeung et al. (2010) have shown that the proportion of cancer stem cells that can be identified in colorectal cancer derived cell lines can vary quite widely, from perhaps $20 \%$ to virtually all cells. Thus, evidence is accumulating that different individual cancers may contain widely differing proportions of CSCs, though the average proportion may differ between different types of tumours, such as breast and colorectal cancers and melanomas.

It is not at all clear that CSCs are necessarily derived from tissue stem cells (Polyak and Hahn, 2006; van Es et al., 2005). In their original three compartment mathematical model, Tomlinson and Bodmer (1995) raised the possibility that cancers may be initiated from transit amplifying cells rather than the tissue stem cells. In their subsequent development of this model, incorporating feedback and using a continuous time model, Johnston et al. (2007a, 2007b) suggested that, while both derivation from the 
tissue stem cell or from the transit amplifying cell may be possible, the ultimate derivation of the CSC is most likely to be traced back to progenitor or transit amplifying cells derived from the tissue stem cell, rather than from the tissue stem cell itself. The aim of this note is to show that very plausible models of a cancer, along the lines of our earlier work, can readily explain substantial variation in the proportion of CSCs versus differentiated cells in a cancer, depending on the balance between the rate of turnover of the CSCs and their rate of differentiation.

\section{Colorectal crypt compartment model}

Johnston et al. (2007a, 2007b) introduced a three compartment continuous time mathematical model of cell turnover in the colorectal crypt that was based on the discrete model of Tomlinson and Bodmer (1995). The cells in a healthy crypt are separated into populations of stem, transit-amplifying and fully differentiated cells, denoted by $N_{0}, N_{1}$ and $N_{2}$, respectively. For simplicity, we ignore the fact that there are three types of differentiated cells in the colorectal crypt. We assume that the stem (transit) cells either die, differentiate or renew at rates $\alpha_{1}, \alpha_{2}$ and $\alpha_{3}$ respectively $\left(\beta_{1}, \beta_{2}\right.$ and $\left.\beta_{3}\right)$, and that the fully differentiated cells eventually die and are shed into the lumen at a rate $\gamma$ (see Fig. 1).

The dynamics of the three cell populations can then be described by a system of three coupled differential equations. The net growth rates of the stem $\left(N_{0}\right)$ and transit amplifying $\left(N_{1}\right)$ cells are, respectively, $\alpha=\alpha_{3}-\alpha_{1}-\alpha_{2}$ and $\beta=\beta_{3}-\beta_{1}-\beta_{2}$. The equilibrium situation in the normal crypt requires that $\alpha=0$, and that $\beta<0$. The initiation and progression of a cancer are determined by genetically or epigenetically based changes in $\alpha$ and $\beta$ rates that disrupt the normal balance of the three types of cells and eventually lead to exponential unlimited growth, when either $\alpha$ or $\beta$ or both exceed 0 . Johnston et al. (2007a, 2007b) showed that plausible models of cancer progression required the introduction of saturating feedback terms, which were introduced by replacing the differentiation rates $\alpha_{2}$ and $\beta_{2}$ by, respectively, the terms $\alpha_{2}+k_{0} N_{0} /\left(1+m_{0} N_{0}\right)$ and $\beta_{2}+k_{1} N_{1} /\left(1+m_{1} N_{1}\right)$ in the three coupled differential equations. The positive constants $k_{0}$ and $k_{1}$ represent the speed of response of the feedback, while the positive constants $m_{0}$ and $m_{1}$ represent feedback saturation.

With this model, as first suggested by Tomlinson and Bodmer (1995), there can be a series of new stable equilibria with increased numbers of stem cells or transit amplifying cells, so long as the net growth rates $\alpha$ or $\beta$ do not exceed, respectively, $k_{0} / m_{0}$ or $k_{1} / m_{1}$. These equilibria correspond to benign or adenomatous stages of tumour progression. Only when either $\alpha$ or $\beta$ exceed their respective limits, will exponential growth representing the full development of a cancer occur. When $\alpha$ first exceeds its limit, the CSCs, which drive the exponential growth of the cancer, are derived from the normal tissue stem cells. However, if $\beta$ first exceeds its limit then the CSCs are derived from the transit amplifying cells. Johnston et al. (2007b) showed that if $m_{0}=0$, so that there is no saturation of the feedback for the stem cells representing, plausibly, greater feedback constraint on them than on the transit amplifying cells, CSCs will always be derived from the transit-amplifying cells. This may be, as we have already mentioned, the more usual situation.

\section{Modelling the exponential growth phase}

Johnston et al. (2007a, 2007b), as discussed above, showed that their model could lead to instability either through growth of the tissue stem cells $\left(N_{0}\right)$ driving proliferation, or through the semidifferentiated cells $\left(N_{1}\right)$ driving growth. We now consider both these cases in turn.

In order to obtain estimates of the proportion of CSCs in a tumour based on the above model, we have to consider the behaviour of the differential equations when $N_{0}, N_{1}$ and $N_{2}$ are very large. It can be shown that the three coupled differential equations describing the model with feedback saturation then approximate to

$\frac{\mathrm{d} N_{0}}{\mathrm{~d} t} \approx\left(\alpha-k_{0} / m_{0}\right) N_{0}$,

$\frac{\mathrm{d} N_{1}}{\mathrm{~d} t} \approx\left(\beta-k_{1} / m_{1}\right) N_{1}+\left(\alpha_{2}+k_{0} / m_{0}\right) N_{0}$,

$\frac{\mathrm{d} N_{2}}{\mathrm{~d} t} \approx-\gamma N_{2}+\left(\beta_{2}+k_{1} / m_{1}\right) N_{1}$

The parameters in these equations now represent the ultimate values reached at the final exponential growth phase of the cancer. These equations can be explicitly solved to obtain a general expression for the limiting ratios of $N_{0}: N_{1}: N_{2}$ (see Supplementary material).

If the CSCs are derived from a normal tissue stem cell, then $N_{0} /\left(N_{0}+N_{1}+N_{2}\right)$ is the proportion of CSCs in the tumour. This proportion can be expressed in the form $1 /(1+P)$, where $P$ is given by (see Supplementary material)

$P=\frac{\alpha_{2}+k_{0} / m_{0}}{\alpha-k_{0} / m_{0}-\beta+k_{1} / m_{1}}\left(1+\frac{\beta_{2}+k_{1} / m_{1}}{\alpha-k_{0} / m_{0}+\gamma}\right)$.

Thus, depending on the value of the various parameters, the proportion of CSCs could be either close to 1 (when $P$ is very small) or near to 0 (when $P$ is very large). In Fig. 2 we illustrate some numerical examples which show how variations in the parameter $P$, or in any of its constituent components, such as $\alpha_{3}$, can allow the proportion of CSCs to vary substantially.

When the CSCs are derived from the transit amplifying cells, $N_{0}$ remains constant and therefore can be ignored in relation to the exponentially increasing values of $N_{1}$ and $N_{2}$. The proportion of CSCs in the tumour is then $N_{1} /\left(N_{1}+N_{2}\right)=1 /(1+P)$, where $P$ is now given by (see Supplementary material)

$P=\frac{\beta_{2}+k_{1} / m_{1}}{\beta-k_{1} / m_{1}+\gamma}$.

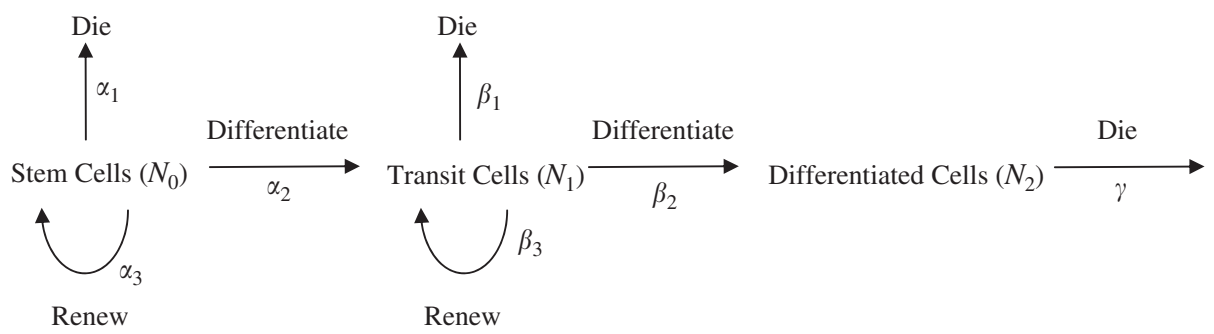

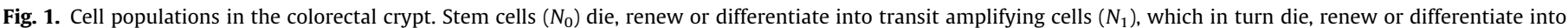
fully differentiated cells $\left(N_{2}\right)$. The parameters $\alpha_{1}, \alpha_{2}, \alpha_{3}, \beta_{1}, \beta_{2}, \beta_{3}$ and $\gamma$ are the rates at which these processes occur. 
a

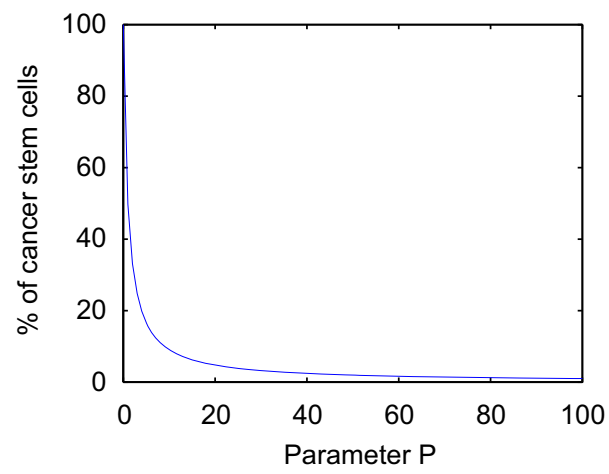

b

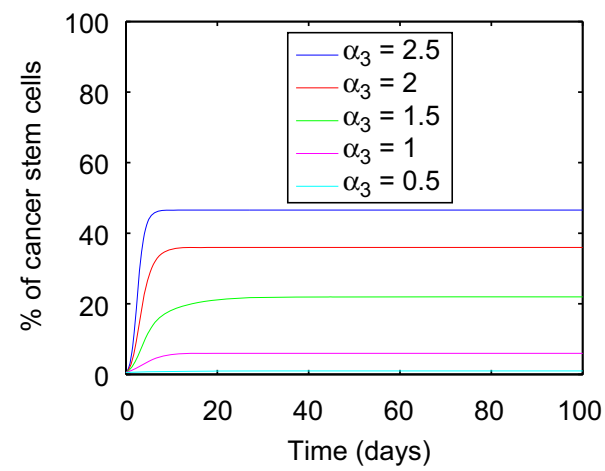

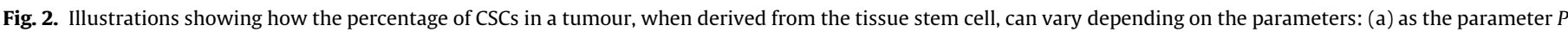

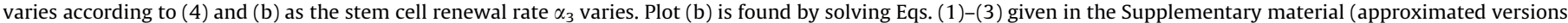

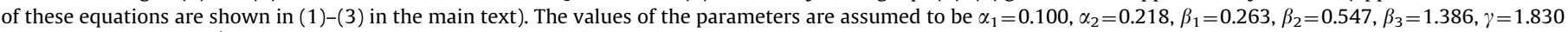
(all measured in days ${ }^{-1}$ ). $k_{0}=0.1, k_{1}=0.01, m_{0}=0.1$ and $m_{1}=0.01$.

When the maximum rate of differentiation of the $\operatorname{CSCs}\left(\beta_{2}+k_{1} / m_{1}\right)$ is small, then $P$ can be near zero and the proportion of CSCs near 1 . On the other hand, when the CSCs can still differentiate well, and their maximum growth rate together with the rate of fully differentiated cell removal $\left(\beta-k_{1} / m_{1}+\gamma\right)$ is small relative to this, $P$ will be larger and therefore the proportion of CSCs smaller. This agrees well with the clinical expectation that the less the propensity of a tumour to differentiate, the more aggressive it is likely to be and the higher the proportion of CSCs it is likely to contain.

\section{Discussion}

Recently, there have been a number of models addressing the control and progression of cancer stem cells (see, for example, Enderling et al., 2009) and of stem cells in general tissue (Lo et al., 2009, and references therein). While a number of models exist for the spatiotemporal dynamics of cells in crypts (see, for example, van Leeuwen et al., 2009 and references therein) or, as in the present model, implicitly including spatial constraints in a temporal model (see, for example, Boman et al., 2001; d'Onofrio and Tomlinson, 2007) to our knowledge, there has been no previous attempt to use mathematical modelling to predict the proportion of CSCs in a tumour.

We have shown that, when our previously analysed model of cell turnover in the colorectal crypt is analysed at the exponential growth phase of a cancer, it is possible to explain almost any proportion of CSCs in a tumour under quite plausible assumptions. This is in marked contrast to the previously widely made assumption that CSCs necessarily constitute a small proportion of the cells in a cancer. Our result is supported by specific experimental evidence at least in melanomas (Quintana et al., 2008), lymphoid and myeloid malignancies (Kelly et al., 2007), and colorectal cancer derived cell lines (Yeung et al., 2010). It is, of course, entirely possible that the average proportion of CSCs in a cancer may vary from one tissue to another. In any case, according to our model, the proportion of CSCs depends on the balance between their rates of growth and differentiation, and this is just as would be expected from clinical and experimental data.

\section{Author contributions}

All authors were involved in setting up the model and interpreting the results, W.F.B. designed the study and M.D.J. carried out the analysis.

\section{Acknowledgements}

We acknowledge the support provided by the funders of the Integrative Biology project: the EPSRC (GR/S720231/01) and IBM. M.D.J. was supported by an EPSRC DTA graduate studentship (Award no. EP/P500397/1) and the Sarah and Nadine Pole Scholarship, which are gratefully acknowledged. W.F.B. was supported by a programme grant from Cancer Research UK and is grateful for helpful discussions with Trevor Yeung and Shaan Gandhi. P.K.M. was partially supported by a Royal Society Wolfson Research Merit Award.

\section{Appendix A. Supplementary material}

Supplementary data associated with this article can be found in the online version at doi:10.1016/j.jtbi.2010.07.031.

\section{References}

Al-Hajj, M., Wicha, M.S., Benito-Hernandez, A., Morrison, S.J., Clarke, M.F., 2003. Prospective identification of tumorigenic breast cancer cells. Proc. Natl. Acad. Sci. USA 100, 3983-3988.

Baker, M., 2008. Cancer stem cells, becoming common. Nature. doi:10.1038 stemcells.2008.153.

Boman, B.M., Fields, J.Z., Bonham-Carter, O., Runquist, O.A., 2001. Computer modeling implicates stem cell overproduction in colon cancer initiation. Cancer Res. 61, 8408-8411.

Cho, R.W., Clarke, M.F., 2008. Recent advances in cancer stem cells. Curr. Opin. Genet. Dev. 18, 48-53.

Clarke, M.F., 2004. Neurobiology: at the root of brain cancer. Nature 432, 281-282.

Dalerba, P., Cho, R.W., Clarke, M.F., 2007a. Cancer stem cells: models and concepts. Annu. Rev. Med. 58, 267-284.

Dalerba, P., et al., 2007b. Phenotypic characterization of human colorectal cancer stem cells. Proc. Natl. Acad. Sci. USA 104, 10158-10163.

d'Onofrio, A., Tomlinson, P.M., 2007. A nonlinear mathematical model of cell turnover, differentiation and tumorigenesis in the intestinal crypt. J. Theor. Biol. 244, 367-374.

Enderling, H., et al., 2009. Paradoxical dependencies of tumor dormancy and progression on basic cell kinetics. Cancer Res. 69 (22), 8814-8821.

Harper, L.J., Piper, K., Common, J., Fortune, F., Mackenzie, I.C., 2007. Stem cell patterns in cell lines derived from head and neck squamous cell carcinoma. J. Oral Pathol. Med. 36, 594-603.

Huntly, B.J.P., Gilliland, D.G., 2005. Summing up cancer stem cells. Nature 435, 1169-1170.

Johnston, M.D., Edwards, C.M., Bodmer, W.F., Maini, P.K., Chapman, S.J., 2007a Mathematical modeling of cell population dynamics in the colonic crypt and in colorectal cancer. Proc. Natl. Acad. Sci. USA 104, 4008-4013.

Johnston, M.D., Edwards, C.M., Bodmer, W.F., Maini, P.K., Chapman, S.J., 2007b. Examples of mathematical modeling: tales from the crypt. Cell Cycle 6, 2106-2112.

Kelly, P.N., Dakic, A., Adams, J.M., Nutt, S.L., Strasser, A., 2007. Tumor growth need not be driven by rare cancer stem cells. Science 317, 337.

Lo, W-C., et al., 2009. Feedback regulation in multistage cell lineages. Math. Biosci. Eng. 6 (1), 59-82. 
Locke, M., Heywood, M., Fawell, S., Mackenzie, I.C., 2005. Retention of intrinsic stem cell hierarchies in carcinoma-derived cell lines. Cancer Res. 65, 8944-8950.

McDonald, S.A.C., Preston, S.L., Lovell, M.J., Wright, N.A., Jankowski, J.A.Z., 2006 Mechanisms of disease: from stem cells to colorectal cancer. Nat. Clin. Pract. Gastroenterol. Hepatol. 3, 267-274.

Mackenzie, I.C., 2005. Retention of stem cell patterns in malignant cell lines. Cell Prolif. 38, 347-355.

Michor, F., et al., 2005. Dynamics of chronic myeloid leukaemia. Nature 435 1267-1270.

O’Brien, C.A., Pollett, A., Gallinger, S., Dick, J.E., 2007. A human colon cancer cell capable of initiating tumour growth in immunodeficient mice. Nature 445, 106-110.

Polyak, K., Hahn, W.C., 2006. Roots and stems: stem cells in cancer. Nat. Med. 12, 296-300.

Quintana, E., et al., 2008. Efficient tumour formation by single human melanoma cells. Nature 456, 593-598.
Reya, T., Morrison, S.J., Clarke, M.F., Weissman, I.L., 2001. Stem cells, cancer, and cancer stem cells. Nature 414, 105-111.

Ricci-Vitiani, L., et al., 2007. Identification and expansion of human colon-cancerinitiating cells. Nature 445, 111-115.

Singh, S.K., et al., 2004. Identification of human brain tumour initiating cells. Nature 432, 396-401.

Tomlinson, I.P., Bodmer, W.F., 1995. Failure of programmed cell death and differentiation as causes of tumors: some simple mathematical models. Proc. Natl. Acad. Sci. USA 92, 11130-11134.

van Es, J.H., et al., 2005. Notch $/ \gamma$-secretase inhibition turns proliferative cells in intestinal crypts and adenomas into goblet cells. Nature 435, 959-963.

van Leeuwen, I.M.M., et al., 2009. An integrative computational model for intestinal tissue renewal. Cell Prolif. 42, 617-636.

Yeung, T.M., Gandhi, S.C., Wilding, J.L., Muschel, R., Bodmer, W.F., 2010. Cancer stem cells from colorectal cancer-derived cell lines. Proc. Natl. Acad. Sci. USA 107, 3722-3727. 